\title{
COEFFICIENT BOUNDS FOR THE INVERSE OF A FUNCTION WITH DERIVATIVE IN $\odot$
}

\author{
RICHARD J. LIBERA AND ELIGIUSZ J. ZLOTKIEWICZ
}

\begin{abstract}
Coefficient bounds for functions with a positive real part are used in a rather novel way to find sharp bounds for the first six coefficients of a function which is inverse to a regular normalized univalent function whose derivative has a positive real part in the unit disk.
\end{abstract}

1. Introduction. The family $\S$ of all functions regular and one-to-one in the open unit disk $\Delta, \Delta=\{z \in \mathbf{C}:|z|<1\}$, consists of functions of the form

$$
f(z)=z+a_{2} z^{2}+a_{3} z^{2}+\cdots, \text { for } z \text { in } \Delta .
$$

These functions have a rich history $[1,2,10,11]$ and the conjecture that the magnitudes of successive coefficients $a_{n}, n=2,3, \ldots$, are bounded by those of the Koebe function, $k(z)=z+2 z^{2}+\cdots+n z^{n}+\cdots$, has received widespread attention (and confirmation only for $n=2,3,4,5,6)$.

The inverse of $f(z)$ has a series expansion in some disk about the origin of the form

$$
\check{f}(w)=w+\gamma_{2} w^{2}+\gamma_{3} w^{3}+\cdots .
$$

It was shown early $[9,11]$ that the inverse of the Koebe function provides the best bound for all $\left|\gamma_{k}\right|$. New proofs of the latter along with unexpected and unusual behavior of the coefficients $\gamma_{k}$ for various subclasses of $\mathcal{S}$ have generated further interest in this problem $[6,7,8,12]$. The purpose of this paper is to examine the early coefficients of (1.2) for a subclass of $\delta$.

As is usually the case we let $\mathscr{P}$ be the family of functions

$$
P(z)=1+c_{1} z+c_{2} z^{2}+\cdots
$$

regular and with $(R$ e $P(z)>0$ for $z$ in $\Delta$. Furthermore we denote by $q$ the class of all functions of form (1.1) defined by

$$
f(z)=\int_{0}^{z} P(\zeta) d \zeta, \quad z \text { in } \Delta,
$$

$P(\zeta)$ being any member of $(P$. It is well known that $(1.4)$ is one-to-one, consequently $q \subset \bar{s}$. For each $f(z)$ in $q, f[\Delta]$, the image of $\Delta$ under $f(z)$, includes the disk, [4],

$$
\{w \in \mathbf{C}:|w|<\log 4-1\},
$$

Received by the editors February 16, 1982

1980 Mathematics Subject Classification. Primary 30C45; Secondary 30C50.

(c) 983 American Mathematical Society $0002-9939 / 82 / 0000-0785 / \$ 02.25$ 
which is also the common region of convergence of series (1.2). Using known bounds on the $c_{k}$ 's, in (1.3), we are able to give precise bounds on $\left|\gamma_{n}\right|$ for $n=2,3,4,5,6$ and, by a different method, an estimate for $\left|\gamma_{n}\right|$ for all $n$. The main results are stated and discussed in $\$ 2$. Proof of the main theorem is given in $\$ 3$.

2. Principal results. First let us observe that the coefficients $\gamma_{k}$ cannot be uniformly bounded over $\mathcal{G}$, since if they were it would be possible to replace the disk in (1.5) by a larger one.

THEOREM 1. If $f(z)$ is in $\mathscr{G}$ and $\check{f}(w)=\sum_{k=0}^{\infty} \gamma_{k} w^{k}$, then

$$
\left|\gamma_{2}\right| \leqslant 1, \quad\left|\gamma_{3}\right| \leqslant \frac{4}{3}, \quad\left|\gamma_{4}\right| \leqslant \frac{13}{6}, \quad\left|\gamma_{5}\right| \leqslant \frac{59}{15} \quad \text { and } \quad\left|\gamma_{6}\right| \leqslant \frac{344}{45}
$$

and these bounds are all best possible.

THEOREM 2. For $f(z)$ as in Theorem 1,

$$
\begin{aligned}
\left|\gamma_{n}\right| \leqslant \frac{1}{\pi n} \int_{0}^{\pi} \frac{d \theta}{\left|1+2 e^{-i \theta} \log \left(1-e^{i \theta}\right)\right|^{n}} & <\frac{1}{n \alpha^{n}}, \\
n & =2,3, \ldots, \text { with } \alpha=\log \left(\frac{4}{e}\right) .
\end{aligned}
$$

The last statement is fairly easy to establish using the subordination principle. From Cauchy's formula for $\gamma_{n}$ along with the relationship between $f(z)$ and $\check{f}(w)$, as was done earlier in [7], we may conclude that

$$
\gamma_{n}=\frac{1}{2 \pi i n} \int_{|z|=r}\left[\frac{z}{f(z)}\right]^{n} \frac{d z}{z^{n}} .
$$

We need a bound on the integrand.

Hallenbeck [4] has shown that under the stated conditions $f(z) / z$ is subordinate to $-1-(2 / z) \log (1-z)$, consequently, $z / f(z)$ is subordinate to the reciprocal $-z /(z+2 \log (1-z))$. This says there is a regular function $w(z),|w(z)| \leqslant|z|$, for $z$ in $\Delta$, with the property that

$$
\frac{z}{f(z)}=\frac{-w(z)}{w(z)+2 \log (1-w(z))} .
$$

Now, combining (2.4) with (2.3) and applying Rogozinski's majorization principle for subordinate functions (see p. 369, [2], for example) enables us to write

$$
\left|\gamma_{n}\right| \leqslant \frac{1}{2 \pi n} \int_{0}^{2 \pi}\left|\frac{1}{r e^{i \theta}+2 \log \left(1-r e^{i \theta}\right)}\right|^{n} d \theta,
$$

from which the first bound in (2.2) follows. The remaining bound is obtained by maximizing the integrand in (2.5).

A comparison of results in Theorems 1 and 2 shows that the bounds in (2.2) cannot be the best possible. However, those in (2.1) are sharp when $f(z)$ corresponds to

$$
P_{0}(z)=\frac{1+z}{1-z}=1+\sum_{k=1}^{\infty} 2 z^{k}
$$


in 9 . Also, $P_{0}(z)$ is a function which shows that the lemmata which follow cannot be improved.

3. Justification of Theorem 1. A function $f(z)$ univalent in a neighborhood of the origin and its inverse satisfy the condition $f\left(\check{f}\left(w^{\prime}\right)\right)=w^{\prime}$, or

$$
w=\check{f}(w)+a_{2}(\check{f}(w))^{2}+a_{3}\left(\check{f}\left(w^{\prime}\right)\right)^{3}+\cdots .
$$

Assuming (1.1) and (1.2). (3.1) yields the equations

$$
\left\{\begin{array}{l}
\gamma_{2}+a_{2}=0, \quad \gamma_{3}+2 a_{2} \gamma_{3}+a_{3}=0 \\
\gamma_{4}+a_{2}\left(\gamma_{2}^{2}+2 \gamma_{3}\right)+3 a_{3} \gamma_{2}+a_{4}=0, \\
\gamma_{5}+a_{2}\left(2 \gamma_{4}+2 \gamma_{2} \gamma_{3}\right)+a_{3}\left(3 \gamma_{3}+3 \gamma_{2}^{2}\right)+4 a_{4} \gamma_{2}+a_{5}=0 . \\
\quad \gamma_{6}+a_{2}\left(2 \gamma_{5}+2 \gamma_{2} \gamma_{4}+\gamma_{3}^{2}\right)+a_{3}\left(6 \gamma_{2} \gamma_{3}+3 \gamma_{4}+\gamma_{2}^{3}\right) \\
\quad+a_{4}\left(6 \gamma_{2}^{2}+4 \gamma_{3}\right)+5 a_{5} \gamma_{2}+a_{6}=0 \\
\text { and } \\
\quad+a_{7}\left(2 \gamma_{6}+2 \gamma_{2} \gamma_{5}+2 \gamma_{3} \gamma_{4}\right)+a_{3}\left(3 \gamma_{5}+6 \gamma_{2} \gamma_{4}+3 \gamma_{3}^{2}+3 \gamma_{2}^{2} \gamma_{3}\right) \\
\quad+a_{4}\left(4 \gamma_{4}+12 \gamma_{2} \gamma_{3}+4 \gamma_{2}^{3}\right)+a_{5}\left(5 \gamma_{3}+10 \gamma_{2}^{2}\right)+6 a_{6} \gamma_{2}+a_{7}=0 .
\end{array}\right.
$$

Because of the relationship between $\varphi$ and $? \%$ we write

$$
a_{k}=\frac{c_{k-1}}{k}, \quad k=2,3, \ldots
$$

using representation (1.3). Combining (3.2) and (3.3) gives

$$
\left\{\begin{array}{l}
2 \gamma_{2}=-c_{1}, \quad 6 \gamma_{3}=3 c_{1}^{2}-2 c_{2}, \\
24 \gamma_{4}=20 c_{1} c_{2}-15 c_{1}^{3}-6 c_{3}, \\
120 \gamma_{5}=90 c_{1} c_{3}+40 c_{2}^{2}+105 c_{1}^{4}-210 c_{1}^{2} c_{2}-24 c_{4}, \\
\text { and } \\
720 \gamma_{6}=504 c_{1} c_{4}+420 c_{2} c_{3}+2520 c_{1}^{3} c_{2}-1260 c_{1}^{3} c_{3} \\
\quad-1120 c_{1} c_{2}^{2}-945 c_{1}^{5}-120 c_{5} .
\end{array}\right.
$$

It is well-known $[10,11]$ that $\left|c_{k}\right| \leqslant 2$ for $k=1,2, \ldots$ hence, we may write $2\left|\gamma_{2}\right|=\left|c_{1}\right| \leqslant 2$. This gives the first result in the theorem. For the other bounds we need additional properties of the coefficients $c_{k}$.

LEMMA 1. If $P(z) \in$ ip and $1 / P(z)=1+\sum_{k=1}^{\infty} c_{k}^{*} z^{k}$, then

$$
\left\{\begin{array}{l}
c_{1}^{*}=c_{1}^{2}-c_{2}, \quad c_{2}^{*}=c_{3}-2 c_{1} c_{2}+c_{1}^{3}, \\
c_{3}^{*}=c_{1}^{4}+c_{2}^{2}+2 c_{1} c_{3}-3 c_{1}^{2} c_{2}-c_{4}, \\
c_{4}^{*}=c_{5}+c_{1}^{5}+3 c_{1} c_{2}^{2}+3 c_{1}^{2} c_{3}-4 c_{1}^{3} c_{2}-2 c_{1} c_{4}-2 c_{2} c_{3}, \\
\text { and } \\
c_{5}^{*}=c_{1}^{6}+6 c_{1}^{2} c_{2}^{2}+4 c_{1}^{3} c_{3}+2 c_{1} c_{5}+2 c_{2} c_{4}+c_{3}^{2} \\
\quad-c_{2}^{3}-5 c_{1}^{4} c_{2}-3 c_{1}^{2} c_{4}-6 c_{1} c_{2} c_{3}-c_{6} ;
\end{array}\right.
$$


and

$$
\left|c_{h}^{*}\right| \leqslant 2, \quad k=1,2,3, \ldots
$$

The last statement follows from the observation that both $P(z)$ and its reciprocal are in $\%$.

Using this lemma and (3.4) gives $6\left|\gamma_{3}\right| \leqslant\left|c_{1}\right|^{2}+2\left|c_{1}^{*}\right| \leqslant 8$ and $24\left|\gamma_{4}\right| \leqslant 6\left|c_{2}^{*}\right|$ $+\left|c_{1}\right|^{3}+8\left|c_{1}\right| \cdot\left|c_{1}^{*}\right| \leqslant 52$ which correspond to the second and third inequalities in (2.1). The last two require additional techniques.

LEMMA 2. The power series for $P(z)$ given in (1.3) converges in $\Delta$ to a function in $(?)$ if and only if the Toeplitz determinants

$$
D_{n}=\left|\begin{array}{ccccc}
2 & c_{1} & c_{2} & \ldots & c_{n} \\
c_{-1} & 2 & c_{1} & \ldots & c_{n-1} \\
\vdots & & & & \\
c_{-n} & c_{-n+1} & c_{-n+2} & \ldots & 2
\end{array}\right|, \quad n=1,2,3, \ldots
$$

and $c_{-h}=\bar{c}_{h}$, are all nonnegative. They are strictly positive except for $P(z)=$ $\sum_{h=1}^{m} \rho_{h} P_{0}\left(e^{i t} k z\right), \rho_{k}>0, t_{k}$ real and $t_{k} \neq t_{j}$ for $k \neq j$ and $\sum_{k=1}^{m} \rho_{k}=1$; in this case $D_{n}>0$ for $n<m-1$ and $D_{n}=0$ for $n \geqslant m$.

This necessary and sufficient condition is due to Caratheodory and Toeplitz and can be found in [3]. The following lemma can be obtained from representation (2.3), $[5]$.

LEMMA 3. For any complex number $\alpha$ and $P(z)$ in $P$,

$$
\max \left|c_{2}-\alpha c_{1}^{2}\right|=2 \max \{1,|2 \alpha-1|\} .
$$

We need the first two lemmas to obtain the bound on $\left|\gamma_{5}\right|$ and all three for $\left|\gamma_{6}\right|$. We may assume without restriction that $c_{1} \geqslant 0$. We begin by rewriting (3.7) for the cases $n=2$ and $n=3$.

$$
D_{2}=\left|\begin{array}{ccc}
2 & c_{1} & c_{2} \\
c_{1} & 2 & c_{1} \\
\bar{c}_{2} & c_{1} & 2
\end{array}\right|=8+2 \Re \mathrm{e}\left\{c_{1}^{2} c_{2}\right\}-2\left|c_{2}\right|^{2}-4 c_{1}^{2} \geqslant 0
$$

which is equivalent to

$$
2 c_{2}=c_{1}^{2}+x\left(4-c_{1}\right)^{2}
$$

for some $x,|x| \leqslant 1$.

Then $D_{3} \geqslant 0$ is equivalent to

$$
\left|\left(4 c_{3}-4 c_{1} c_{2}+c_{1}^{3}\right)\left(4-c_{1}^{2}\right)+c_{1}\left(2 c_{2}-c_{1}^{2}\right)^{2}\right| \leqslant 2\left(4-c_{1}^{2}\right)^{2}-2\left|2 c_{2}-c_{1}^{2}\right|^{2}
$$

and this, with (3.9), provides the relation

(3.10) $4 c_{3}=c_{1}^{3}+2\left(4-c_{1}^{2}\right) c_{1} x-c_{1}\left(4-c_{1}^{2}\right) x^{2}+2\left(4-c_{1}^{2}\right)\left(1-|x|^{2}\right) z$,

for some value of $z,|z| \leqslant 1$. 
By proper rearrangement and application of the triangle inequality to the fourth member of (3.4) we get

$$
120\left|\gamma_{5}\right| \leqslant 24\left|c_{3}^{*}\right|+42\left|c_{1}\right| \cdot\left|c_{2}^{*}\right|+\left|16 c_{2}^{2}+39 c_{1}^{4}-54 c_{1}^{2} c_{2}\right| ;
$$

then the bounds given above and (3.9) make it possible to write

$$
\begin{aligned}
120\left|\gamma_{5}\right| & \leqslant 216+\left|16 c_{2}^{2}+39 c_{1}^{4}-54 c_{1}^{2} c_{2}\right| \\
& =216+\left|16 c_{1}^{4}-19 c_{1}^{2}\left(4-c_{1}^{2}\right) x+4\left(16-8 c_{1}^{2}+c_{1}^{4}\right) x^{2}\right| \\
& \leqslant 216+\left(64+44 c_{1}^{2}+c_{1}^{4}\right) \leqslant 216+256=472
\end{aligned}
$$

from which we conclude that $\left|\gamma_{5}\right| \leqslant 59 / 15$.

Applying similar procedures to $\gamma_{6}$ we have

$$
\left\{\begin{array}{c}
720\left|\gamma_{6}\right| \leqslant 120\left|c_{5}^{*}\right|+264\left|c_{1}\right| \cdot\left|c_{4}^{*}\right|+372\left|c_{1}\right|^{2} \cdot\left|c_{3}^{*}\right|+B \leqslant 4272+B \\
B=\left|504 c_{1}^{3} c_{2}+180 c_{2} c_{3}-496 c_{1} c_{2}^{2}-189 c_{1}^{5}\right| .
\end{array}\right.
$$

To generate a sharp upper bound for $B$ we find it convenient to consider two cases depending on choices for $c_{1}$.

Therefore, we assume $0 \leqslant c_{1} \leqslant \frac{1}{2}$. Then

$$
B \leqslant\left|496 c_{1} c_{2}^{2}-180 c_{2} c_{3}\right|+504 c_{1}^{3}\left|c_{2}-\frac{189}{504} c_{1}^{2}\right|,
$$

and as a consequence of Lemma 3 we find that 126 is an upper bound for the second term in (3.13). Now, using the bound $\left|c_{2}\right| \leqslant 2$ and (3.9) and (3.10), we write

$$
\begin{aligned}
& \left|496 c_{1} c_{2}^{2}-180 c_{2} c_{3}\right| \leqslant 2\left|496 c_{1} c_{2}-180 c_{3}\right| \\
& \leqslant 2\left[203 c_{1}^{3}+\left(4-c_{1}^{2}\right)\left(90+158 c_{1} \rho-45\left(2-c_{1}\right) \rho^{2}\right)\right],
\end{aligned}
$$

with $\rho=|x|, \rho<1$. This second degree polynomial in $\rho$ assumes its maximum when $\rho=79 c_{1} / 45\left(2-c_{1}\right)$ and in this case we find that the last bound in (3.14) can be replaced by

$$
2\left[360+\frac{(124)(34)(2)}{45} c_{1}^{2}+\frac{15,376}{45} c_{1}^{3}\right] \leqslant 814 .
$$

Now, this together with the above bound, shows that $720\left|\gamma_{6}\right| \leqslant 4272+814+126$ $=5212$, which implies the conclusion when $0 \leqslant c_{1} \leqslant \frac{1}{2}$.

Returning again to (3.9), (3.10) and (3.12), we write

$$
\begin{aligned}
2 B=\mid 77 c_{1}^{5}-143 c_{1}^{3}\left(4-c_{1}^{2}\right) x+c_{1}\left(4-c_{1}^{2}\right)\left(632-113 c_{1}^{2}\right) x^{2} \\
\quad+45 c_{1}\left(4-c_{1}^{2}\right)^{2} x^{3}+90\left(4-c_{1}^{2}\right)\left(1-|x|^{2}\right)\left(c_{1}^{2}+\left(4-c_{1}^{2}\right) x\right) z \mid \\
\leqslant\left[77 c_{1}^{5}+90 c_{1}^{2}\left(4-c_{1}^{2}\right)\right]+\left(4-c_{1}^{2}\right) \\
\quad \cdot\left\{\left[143 c_{1}^{3}-90 c_{1}^{2}+360\right] \rho+\left[632 c_{1}-90 c_{1}^{2}-113 c_{1}^{3}\right] \rho^{2}\right. \\
\left.-45\left(4-c_{1}^{2}\right)\left(2-c_{1}\right) \rho^{3}\right\},
\end{aligned}
$$


by letting $\rho=|x|$ and invoking the triangle inequality. If we let $F(\rho)$ denote the last polynomial, then

$$
\begin{array}{r}
F^{\prime}(\rho)=\left(4-c_{1}^{2}\right)\left\{\left[143 c_{1}^{3}-90 c_{1}^{2}+360\right]+\left[1264 c_{1}-180 c_{1}^{2}-226 c_{1}^{3}\right] \rho\right. \\
\left.-135\left(4-c_{1}^{2}\right)\left(2-c_{1}\right) \rho^{2}\right\} .
\end{array}
$$

We wish to show $F^{\prime}(\rho)>0$, for $0 \leqslant \rho \leqslant 1$, and conclude the maximum in (3.15) occurs when $\rho=1$.

For $\frac{1}{2} \leqslant c_{1} \leqslant 2$, the coefficient of $\rho^{2}$ in (3.16) is negative and both $F^{\prime}(0)$ and $F^{\prime}(1)$ are negative; hence, because of the concavity of the quadratic, we conclude that $F^{\prime}(\rho)>0$ for $0 \leqslant \rho \leqslant 1$ and that $F(\rho)$ assumes its maximum at 1 . With $\rho=1$, (3.15) becomes

$$
2 B \leqslant 3248 c_{1}-992 c_{1}^{3}+132 c_{1}^{5} .
$$

The derivative of the right side of (3.17) for $\frac{1}{2} \leqslant c_{1} \leqslant 2$ is positive and the maximum occurs at 2 . Consequently, $2 B \leqslant 2(1232)$ and $720\left|\gamma_{6}\right| \leqslant 4272+1232=5504$ which is equivalent to the bound of the theorem. (Note. The choice of $\frac{1}{2}$ for the cases discussed above is one of convenience rather than necessity.)

The bounds in Theorem 1 are made sharp by the function $\check{f}_{0}(w)$ which corresponds to $f_{0}(z)=-z-2 \log (1-z)$ which is obtained by integrating (2.16). If we let $\check{f}_{0}(w)=w+\sum_{k=1}^{\infty} B_{k} w^{k}$, then we find that the recursion formula

$$
(k+1) B_{k+1}+B_{k}+\sum_{j=1}^{k}(k+1-j) B_{j} B_{k+1-j}=0
$$

holds for $k=1,2, \ldots$, and $B_{1}=1$. Computation gives $B_{2}=-1, B_{3}=4 / 3, B_{4}=$ $-13 / 6, B_{5}=59 / 15$ and $B_{6}=-344 / 45$. It is quite likely that $\check{f}_{0}(w)$ gives the sharp upper bounds for other (perhaps even all) coefficients, but we were unable to show this. It may be possible to utilize techniques like those used above for coefficients of terms of higher degree, but the computations and technical aspects of doing so appear exceedingly formidable.

Finally, let us remark on the nature of the coefficient problems for $f(z)$ and $\check{f}(w)$ for $\delta$ and some of its subclasses. As is shown in $[\mathbf{1}, 2,6,7,8,9,12]$, it appears that for 5 and many of its subclasses the coefficient problem for one of either $f(z)$ or $\check{f}(w)$ is relatively straightforward but extremely difficult for the other. The class of starlike functions is free of this "duality", but some of its subclasses are not [7].

ACKNOWLEDGEMENTS. Some of this work was done while the first author was either at FUB (Berlin) or UMCS (Lublin) under support of DAAD, NAS or PAN and concluded while the second author was a Visiting Professor at the University of Delaware.

\section{REFERENCES}

1. P. L. Duren, Coefficients of univalent functions, Bull. Amer. Math. Soc. 83 (1977), 891-911

2. (i. M. Goluzin, Geometric theory of functions of a complex variable. Transl. Math. Mono. vol. 26. Amer. Math. Soc., Providence, R. I., 1969.

3. U. (irenander and (3. Szegö. Toeplitz forms and their applications. Univ. of California Press. Berkeley and Los Angeles, 1958. 
4. D. J. Hallenbeck, Convex hulls and extreme points of some families of univalent functions. Trans. Amer. Math. Soc. 192 (1974), 285-292.

5. F. Keough and E. Merkes, $A$ coefficient inequality for certain classes of analytic functions, Proc. Amer. Math. Soc. 20 (1969), 8-12.

6. W. E. Kirwan and G. Schober, Inverse coefficients for functions of hounded houndary rotation, J Analyse Math. 36 (1979), 167-178.

7. J. (j. Krzyz, R. J. Libera and E. J. Zlotkiewicz, Coefficients of inverses of regular starlike functions, Ann. Univ. Mariae Curie-Skłodowska Sect. A (to appear).

8. R. J. Libera and E. J. Zlotkiewicz, Early coefficients of the inverse of a regular convex function, Proc. Amer. Math. Soc. 85 (1982), 225-230.

9. K. Löwner, Untersuchungen über schlichte abbildungen des einheitskreises, Math. Ann. 89 (1923), 103-121

10. Z. Nehari, Conformal mapping, McGraw-Hill, New York. 1952.

11. Ch. Pommerenke, Univalent functions, Vandenhoeck and Ruprecht, Göttingen, 1975.

12. G. Schober, Coefficient estimates for inverses of schlicht functions, Aspects of Contemporary Complex Analysis, Academic Press, New York, 1980, pp. 503-513.

Department of Mathematical Sciences, University of Delaware, Newark. Delaware 19711

Instytut Matematyki, UMCS, Ul. Nowotki 10, 20-031 Lublin, Poland 